\title{
Nuclear depletion of RNA-binding protein ELAVL3 (HuC) in sporadic and familial amyotrophic lateral sclerosis
}

\author{
Sandra Diaz-Garcia ${ }^{1}$ - Vivian I. Ko ${ }^{1} \cdot$ Sonia Vazquez-Sanchez ${ }^{2,3} \cdot$ Ruth Chia $^{4}$ - Olubankole Aladesuyi Arogundade ${ }^{1}$. \\ Maria J. Rodriguez ${ }^{1} \cdot$ Bryan J. Traynor ${ }^{4}$. Don Cleveland ${ }^{2,3} \cdot$ John Ravits ${ }^{1}$ (I)
}

Received: 27 May 2021 / Revised: 20 September 2021 / Accepted: 21 September 2021 / Published online: 7 October 2021

(c) The Author(s) 2021

\begin{abstract}
Amyotrophic lateral sclerosis is a progressive fatal neurodegenerative disease caused by loss of motor neurons and characterized neuropathologically in almost all cases by nuclear depletion and cytoplasmic aggregation of TDP-43, a nuclear RNA-binding protein (RBP). We identified ELAVL3 as one of the most downregulated genes in our transcriptome profiles of laser captured microdissection of motor neurons from sporadic ALS nervous systems and the most dysregulated of all RBPs. Neuropathological characterizations showed ELAVL3 nuclear depletion in a great percentage of remnant motor neurons, sometimes accompanied by cytoplasmic accumulations. These abnormalities were common in sporadic cases with and without intermediate expansions in ATXN2 and familial cases carrying mutations in C9orf72 and SOD1. Depletion of ELAVL3 occurred at both the RNA and protein levels and a short protein isoform was identified, but it is not related to a TDP-43-dependent cryptic exon in intron 3. Strikingly, ELAVL3 abnormalities were more frequent than TDP-43 abnormalities and occurred in motor neurons still with normal nuclear TDP-43 present, but all neurons with abnormal TDP-43 also had abnormal ELAVL3. In a neuron-like cell culture model using SH-SY5Y cells, ELAVL3 mislocalization occurred weeks before TDP-43 abnormalities were seen. We interrogated genetic databases, but did not identify association of ELAVL3 genetic structure with ALS. Taken together, these findings suggest that ELAVL3 is an important RBP in ALS pathogenesis acquired early and the neuropathological data suggest that it is involved by loss of function rather than cytoplasmic toxicity.
\end{abstract}

Keywords ALS - Neurodegeneration · ELAVL3 - TDP-43 - RNA-binding proteins $\cdot$ Loss of function · Nuclear depletion · C9orf72 $\cdot$ SOD1 $\cdot$ ATXN2

\section{Abbreviations}

ALS Amyotrophic lateral sclerosis

ARES AU-rich elements

CNS Central nervous system
fALS Familial amyotrophic lateral sclerosis

MN Motor neuron

nELAVLs Neuronal ELAVLs

NLS Nuclear localization signal
John Ravits

jravits@ucsd.edu

Sandra Diaz-Garcia

sdiazgarcia@health.ucsd.edu

Vivian I. Ko

vko@health.ucsd.edu

Sonia Vazquez-Sanchez

soniavazquez-sanchez@health.ucsd.edu

Ruth Chia

ruth.chia@nih.gov

Olubankole Aladesuyi Arogundade

oaladesu@health.ucsd.edu

Maria J. Rodriguez

mjrodriguez@health.ucsd.edu
Bryan J. Traynor

traynorb@mail.nih.gov

Don Cleveland

dcleveland@health.ucsd.edu

Department of Neurosciences, University of California, San Diego, La Jolla, CA 92093-0670, USA

2 Department of Cellular and Molecular Medicine, University of California, San Diego, La Jolla, CA 92093-0670, USA

3 Ludwig Institute for Cancer Research, University of California at San Diego, La Jolla, CA, USA

4 Laboratory of Neurogenetics, National Institute on Aging, National Institutes of Health, Bethesda, MD 20892-3707, USA 
RBP RNA-binding protein

sALS Sporadic amyotrophic lateral sclerosis

3'UTR 3'-Untranslated region

\section{Introduction}

Amyotrophic lateral sclerosis (ALS) is a fatal neurodegenerative disease characterized by adult-onset progressive motor impairments from degeneration of upper and lower motor neurons [5, 28, 58]. Ninety percent of cases are sporadic (sALS) and their causes are unknown. Ten percent of cases are familial (fALS), and over 65 genes have been identified, the most common genes being C9orf72 and SOD1 [38, 54]. The signature neuropathological hallmarks of ALS are nuclear depletion, mislocalization, and cytoplasmic aggregation of TDP-43, and these are characteristic in 97\% of cases-essentially all sALS and most fALS except for SOD1 and FUS-mutated ALS [37]. The discovery of TDP-43, a nuclear RNA-binding protein (RBP), identified the importance of RNA biology in ALS pathogenesis and led to identification of several other RBPs including FUS [2, 33, 44], TAF15 [27, 55], hnRNPA1 [13, 16, 17, 26, 61], hnRNPA2B1 [29], EWS [42], and MATR3 [23]. It is unclear how these RBPs contribute to pathogenesis and the debate has focused on loss of their nuclear functions highlighted neuropathologically by nuclear depletion and cytoplasm toxicity highlighted neuropathologically by cytoplasmic accumulation of inclusions and aggregations [4, 9, 18, 54, 57].

While ALS is a disease that specifically affects motor neurons in the central nervous system, many of the altered RBPs involved in ALS are ubiquitously expressed. ELAVL (or $\mathrm{Hu}$ ) proteins are RBPs highly expressed in neurons and fundamental for the central nervous system development. They were discovered as antigens from patients with paraneoplastic neurological syndromes [10, 30]. Four ELAVL family members-ELAVL1 (HuA or HuR), ELAVL2 (HuB), ELAVL3 (HuC), and ELAVL4 (HuD) - have been defined. ELAVL1 is ubiquitously expressed in many cell types, and ELAVL2, ELAVL3, and ELAVL4 are specifically expressed in peripheral and central neurons throughout development and therefore are sometimes referred to as neuronal ELAVLs (nELAVLs) [20, 22, 47, 53]. ELAVL proteins modulate mRNA stability, splicing, and translational efficiency by binding AU-rich elements (ARES) or alternative polyadenylation sites $[14,19,22,36,60]$. The ELAVL proteins are involved with neuronal, axonal, and synaptic structure including maturation, differentiation, maintenance, and survival $[21,22,24,25,34,45$, $46,51,60]$. While the recognition of the role of nELAVLs proteins in general and ELAVL3 in particular in neurological diseases has mainly focused on paraneoplastic neurological syndromes, they have also been previously implicated in ALS pathogenesis [8, 10, 21, 30, 40].

Because of the importance of RBPs in ALS pathogenesis, we analyzed RBPs in our two independent studies profiling transcriptional changes in sALS laser captured motor neurons $[32,48]$. We identified ELAVL3 because of the degree and statistical significance of the downregulation, the reproducibility of its downregulation in two independent data sets, its exclusively neuronal expression, and its relatively underrecognized role in ALS. We performed extensive neuropathological characterizations and comparisons to TDP-43 pathology. We found several abnormalities in ELAVL3: reduced RNA levels, nuclear depletion, scarce cytoplasmic aggregation, depletion of normal full-length protein, and a short isoform in reduced quantity. The abnormalities were even more prevalent than TDP-43 abnormalities and, in addition, all neurons with abnormal TDP-43 also had abnormal ELAVL3 but not vice versa. Surprisingly, ELAVL3 was also abnormal in SOD1 mutant ALS, where TDP-43 is known to remain normal. ELAVL3 abnormalities were observed upstream of TDP43 abnormalities in an in vitro cell model. Interrogation of genetic structure did not reveal significant susceptibility. Thus, ELAVL3 is an important RBP that is upstream in ALS pathogenesis.

\section{Materials and methods}

\section{CNS tissues}

Human tissues were obtained from the UCSD ALS tissue repository that was created following HIPAA-compliant informed consent procedures approved by Institutional Review Boards (either Benaroya Research Institute, Seattle, WA IRB\# 10058 or University of California San Diego, San Diego, CA IRB\# 120056). Spinal cord and brain tissues were acquired using a short-postmortem interval acquisition protocol usually under $6 \mathrm{~h}$. Tissues were immediately dissected in the autopsy suite, placed in labeled cassettes, and fixed in neutral buffered formalin for at least 2 weeks before being dissected and paraffin-embedded for indefinite storage. For this study, we evaluated from 6 control cases, 31 sALS cases, 5 C9orf72 cases, and 4 SOD1 (Supplementary Table 1).

\section{CNS region and neuronal identification}

Motor neurons were identified by their large size, multipolar cytoplasm, presence of lipofuscin, and large nucleus with a less dense DAPI staining in IF studies. In spinal cord, we examined motor neurons in Rexed lamina IX of anterior 
horn in cervical, thoracic, and lumbar sections. In the cortex, we examined Betz cells in layer $\mathrm{V}$ of the cortex. For quantifications, we chose paraffin blocks that we knew had abundant neurons as revealed by standard H\&E light microscopy and previous studies revealing abundant TDP-43 pathology.

\section{Immunohistochemistry (IHC) and immunofluorescence (IF)}

Tissue sections were cut from blocks of formalin-fixed paraffin-embedded ALS tissue, obtained from the UCSD ALS bank collection. $6 \mu \mathrm{m}$-thick tissue sections were deparaffinized through histology grade CitriSolv (two times for 15 min each) and a graded alcohol series [100, 90, 70, and $50 \%$ ethanol ( $\mathrm{vol} / \mathrm{vol})$ for $5 \mathrm{~min}$ each]. Then, we dipped the slides in de-ionized water, permeabilized for $20 \mathrm{~min}$ in $1 \times$ PBS with $0.2 \%$ Triton $\mathrm{X}-100$, and performed antigen retrieval with $1 \%$ Tris-based (Vector \# H-3301) in a pressure cooker at $120{ }^{\circ} \mathrm{C}$ for $20 \mathrm{~min}$. Sections were further blocked with 2\% Fetal Bovine Serum (vol/vol, Atlanta Biologicals S11150) and incubated with the primary antibody. Primary antibodies were ELAVL3 (\#55047-1-AP from Proteintech (lot number 09000021 was specific for biochemistry) and LS-C408905-50 from LsBio), TDP-43 mouse (\#DR1075-100UG, 1:500; calbiochem), TDP-43 Rabbit (\#10782-2-AP Rabbit Proteintech), HuR-Antibody (\#119101-AP, Proteintech), ELAVL4 (\#24992-1-AP Proteintech), ELAVL2 (\#67097-1-AP Proteintech), and Ubiquitin (Ubi-1 ab7254, Abcam). The antibodies for ELAVL1 and ELAVL3 were previously validated by $\mathrm{KO}$ studies $[35,56,59,62]$. ELAVL2 and ELAVL4 antibodies were validated by protein BLAST alignment demonstrating unique protein sequences. The ELAVL antigen sequences against which the antibodies were made are in Supplementary Table 2. These were incubated overnight at $4{ }^{\circ} \mathrm{C}$. For IHC, we quenched with $0.6 \%$ $\mathrm{H} 2 \mathrm{O} 2$ in Methanol for 15 min before the permeabilization step and continue with the protocol. For both IF and IHC protocols, after incubation with primary antibody, sections were washed four times in $1 \times \mathrm{PBS}$ for $5 \mathrm{~min}$ and blocked with 2\% Normal Donkey Serum (Millipore S30-100 ml) before incubation with secondary antibodies in $1 \times \mathrm{PBS}, 2 \%$ Normal Donkey Serum (vol/vol) for $1 \mathrm{~h}$ at room temperature. For detection of primary antibodies using IF, we used donkey anti-rabbit, anti-mouse Alexa-488, Cy3 conjugated antibodies (Jackson ImmunoResearch) and goat anti-chicken Alexa 633 conjugated antibody (Invitrogen A-21103) at a 1:500 dilution. The slides were then incubated with DAPI $(1 \mu \mathrm{g} / \mathrm{ml})$ followed by a PBS wash. To reduce autofluorescence noise, quenching with $0.1 \%$ Sudan Black in $70 \% \mathrm{EtOH}$ for $15 \mathrm{~s}$ was applied prior to coverslip mounting with ProLong Gold Antifade Mountant with DAPI media (Invitrogen). For IHC after blocked with 2\% Normal Donkey Serum, we incubated with ImmPRESS ${ }^{\circledR}$ HRP Horse Anti-Rabbit
IgG Polymer Detection Kit, Peroxidase (MP-7401 Vector) and developed the antibody with Vector ${ }^{\circledR}$ NovaRED ${ }^{\circledR}$ Substrate Kit, Peroxidase (HRP) (SK-4800 Vector) for 1-2 min until the color was noticed.

\section{Imaging, digital processing, and semiquantitative analysis}

Sections from blocks of the spinal cord and motor cortex were stained with the antibodies described above. We analyzed two sections of $6 \mu \mathrm{m}$ per patient. A semiquantitative assessment of RBP nuclear depletion and mislocalization or decreased expression in ALS tissues was performed taking digital photographs using the Nanozoomer at $40 \mathrm{X}$ and visualizing under $60 \times$ objective in either Nikon confocal A1/LANA system or Leica SP8 confocal systems at UCSD's Microscopy Core facility. Depending on the degree of neurodegeneration of each tissue, we counted as many motor neurons as could be identified as described above. Once neurons were identified, motor neurons were scored based on the presence or absence of nuclear ELAVL3 and TDP-43, being either present or depleted. For illustrations, images were taken at a resolution of $1024 \times 1024$ pixels with a $60 \times$ objective and between 15 and $20 \mathrm{z}$ stack images $(0.25-0.50 \mu \mathrm{m}$ per stack) were collected per frame, and the maximum intensity projection was used to construct the final image series using Fiji software. The .tif files were loaded into Photoshop, where the RGB channels were split. Once the figure was built, each individual channel was inverted to facilitate visualization. The following criteria were used in scoring cytoplasmic accumulations: inclusions were small dot-like, round, diffusely scattered speckles that are relatively uniformly distributed; aggregates were discrete, dense, long, fibrillary, or striae-like (or thread-like) accumulations; and cytoplasmic inclusion bodies were large, round, dense deposits in the cytoplasm present in low number, usually only one per cell.

\section{Cell culture}

Neuroblastoma SH-SY5Y TDP-43-GFP line was obtained from Cleveland Lab. All cells were maintained in highglucose Dulbecco's modified Eagle's medium (DMEM) (Gibco, USA), supplemented with 10\% FBS (Gibco, USA), $2 \mathrm{mM}$ L-glutamine (Lonza, cat. no. BE12-719F), 100 units/ $\mathrm{ml}$ penicillin, and $100 \mu \mathrm{g} / \mathrm{ml}$ streptomycin, in a humidified atmosphere of $5 \%$ carbon dioxide at $37{ }^{\circ} \mathrm{C}$. SH-SY5Y TDP-43EGFP cells were plated on 8-well chamber (Ibidi) at 25,000 cells per well. After $24 \mathrm{~h}$, cells were arrested in G1 with Palbociclib (Apexbio) and maintained during the whole length of the experiment. Cell culture media were changed every 3 days. In all the experiments, the SH-SY5Y control cells and the experimental were plated and maintained in the 
same culture time and media conditions with Palbociclib, without and with rapamycin respectively. $10 \mathrm{nM}$ of Rapamycin was diluted in $100 \mu \mathrm{l}$ of DMSO to use as stressor. Rapamycin was used at a final concentration of $5 \mu \mathrm{M}$ in the media to stress the cells. Rapamycin was maintained in the media for 4 weeks. Cells were fixed with 4\% PFA in PBS for $10 \mathrm{~min}$ at room temperature. After two washes with PBS, cells were permeabilized and blocked with blocking solution (0.1\% Triton, $2 \%$ BSA in PBS) for $1 \mathrm{~h}$ at room temperature. Cells were then incubated overnight with the primary antibody in PBS/0.3\% Tween 20. The primary antibodies used for staining were: anti-ELAVL3 (ab56574, Abcam; 1:1000) at day 1 and after 1,2, and 4 weeks after rapamycin application. After three washes with PBS, the cells were subsequently incubated with fluorescently labeled secondary antibodies diluted at 1:500 in $1 \times \mathrm{PBS}$ for $1 \mathrm{~h}$ at room temperature. After three washes with $1 \times \mathrm{PBS}$, a secondary antibody (anti-rabbit-555 from Jackson) was applied for $1 \mathrm{~h}$. We washed the cells three times for 5 min each with $1 \times$ PBS and mounted with ProLong Gold Antifade Mountant with DAPI media (Invitrogen) to preserve the cells for imaging. SHSY5Y cell imaging was performed on a Ti1 Nikon microscope with a C2 confocal camera at 60-100 $\times$ magnification.

\section{Immunoblotting}

To characterize ELAV3 expression in spinal cord samples, frozen tissue from donors and patients (Supplementary Table 1) was weighted and homogenized in ice-cold N-PER Neuronal Protein Extraction Reagent (Thermofisher \# 87792) with protease and phosphatase inhibitors in $1 \mathrm{ml}$ per $100 \mathrm{mg}$ ratio. The samples were spun down and the supernatants were subjected to Pierce ${ }^{\mathrm{TM}}$ BCA Protein Assay (Thermofisher \#23225). Samples were boiled with Laemmli sample buffer for $10 \mathrm{~min}$ at $90{ }^{\circ} \mathrm{C}$, and to normalize the total amount of proteins among samples, $35 \mu \mathrm{g}$ were loaded in SDS-PAGE (12\% $1 \mathrm{~mm}$ acrylamide gel with 2,2,2-Trichloroethanol), and transferred into Nitrocellulose membranes (Bio-Rad \# 1704159) with Trans-Blot Turbo Transfer System (Bio-Rad\# 1704150) (7 min, 25 V, 2.5 A). Membranes were blocked using iBind ${ }^{\mathrm{TM}}$ Flex Fluorescent Detection (Invitrogen \# SLF2019) and were incubated with primary (1:500 ELAVL3 (\#55047-1-AP Proteintech),1:5000 GAPDH (\#2794431, Millipore), 1:500 TDP-43 (12892-1-AP Proteintech), 1:1000 TUJ1 (\#801202 Biolegend) and secondary antibodies (1:2000 IRDye 800CW Goat anti-Rabbit IgG \# 926-32211 1:2000 IRDye 680RD Goat anti-Mouse IgG \# 926-68070, LI-COR) using iBind ${ }^{\mathrm{TM}}$ Flex Western System (Invitrogen \# SLF2000). NewBlot ${ }^{\mathrm{TM}}$ Nitro Stripping \# 928-40030 LI-COR was use as a stripping buffer between primary antibody incubation when needed. The images were acquired with an Odyssey ${ }^{\circledR} \mathrm{Fc}$ Imaging System from
Li-COR. ImageStudioLite method was used to measure the signal intensity.

\section{Animals}

SOD1 G93A and their littermates $(\mathrm{C} 57 \mathrm{BL} / 6 \times \mathrm{SJL}) \mathrm{F} 1$ (https://www.jax.org/strain/002726), TARDBP tg(tetOTARDBP) mice and their littermates $(\mathrm{C} 57 \mathrm{BL} / 6 \mathrm{~J} \times \mathrm{C} 3 \mathrm{H} / \mathrm{HeJ})$ F1 (https://www.jax.org/strain/016841), and C9ORF72 mice and their littermates $(\mathrm{C} 57 \mathrm{BL} / 6 \times \mathrm{C} 3 \mathrm{H})(\mathrm{https} / / / \mathrm{www} . j a x . o r g /$ strain/030581) were studied.

\section{Gene-specific common variant association and gene-based rare variant analyses}

Individual genotyped data consisted of 10,599 cases and 42,279 controls from Nicolas et al. [43]. Control sample data came from the dbGaP webportal (accession numbers: phs000001, phs000007, phs000101, phs000187, phs000196, phs000248, phs000292, phs000304, phs000315, phs000368, phs000394, phs000397, phs000404, phs000421, phs000428, phs000454, phs000615, phs000675, phs000801, and phs000869). Samples were processed using a standard quality control pipeline [43]. Sample exclusion criteria were: (1) excessive heterozygosity rate (exceeding $\pm 0 \cdot 15 \mathrm{~F}$-statistic), a measure of DNA contamination, (2) low call rate $(\leq 95 \%)$, (3) discordance between reported sex and genotypic sex, (4) duplicate samples (determined by pi-hat statistics $>0.8$ ), and (5) non-European ancestry based on principal components analysis compared to the HapMap 3 Genome Reference Panel. Related samples (defined as having a pi-hat $>0.125$ ) were included for imputation, but one pair member was removed before the association testing.

Variant exclusion criteria were: (1) monomorphic or palindromic SNPs; (2) variants that showed non-random missingness between cases and controls ( $p$ value $\left.\leq 1.0 \times 10^{-4}\right)$; (3) variants with haplotype-based non-random missingness $(p$ value $\left.\leq 1.0 \times 10^{-4}\right) ;(4)$ variants with an overall missingness rate of $\geq 5.0 \%$; (5) non-autosomal variants (X, Y, and mitochondrial chromosomes); and (6) variants that significantly departed from Hardy-Weinberg equilibrium in the control cohort $\left(p\right.$ value $\left.\leq 1.0 \times 10^{-10}\right)$.

Imputation was performed against the Haplotype Reference Consortium (HRC 2016) reference panel (hg19) via minimac 4 using data phased by Eagle v2.4 provided by Michigan Imputation Server (https://imputationserver.sph. umich.edu/). Only variants in ELAVL1,2,3, and 4 genes with imputation quality score (R2) $>0.3$ and minor allele frequency $>0.01$ were included for association analysis. Covariates that were adjusted for to account for non-disease related cohort stratification were sex, age at disease onset, PC1, PC2, PC3, PC4, PC6, PC7, and PC8. Regional plots 
for each gene were generated using LocusZoom (http://locus zoom.org/).

Sequence kernel association test-optimized (SKAT-O) analysis was performed on combined missense and loss-offunction mutations present in ELAVL1,2,3, and 4 genes. The analysis collapses all rare variants and tests for difference in the aggregated burden of rare-coding variants between cases and controls. Variants were annotated using ANNOVAR (v.2018-04/16) prior to association testing using RVTESTS (v.2.1.0). Variants were included in the analysis if the minor allele frequency was $<1 \%$ and minor allele count was $\geq 3$. The Bonferroni threshold for genome-wide significance for missense category was $5.11 \times 10^{-6}(0.05 / 9790$ autosomal genes tested), and for missense and loss-of-function category was $3.60 \times 10^{-6}(0.05 / 13,907$ autosomal genes tested $)$.

\section{Statistical analyses}

Data were analyzed using GraphPad Prism version 7.0a and are expressed as mean \pm standard error of the mean. Significance was assessed using the Student $t$ test-non-parametric Mann-Whitney test, and $p$ values less than 0.05 were considered significant. Chi-square tests used an online calculator (https://www.socscistatistics.com/tests/).

\section{Results}

\section{Identification of ELAVL3 as a candidate RBP in SALS pathogenesis}

We reviewed our previously published transcriptomic data obtained from laser captured spinal motor neurons in sALS nervous systems [32, 48]. We focused our attention on candidate RBPs and identified three that were abnormally expressed in both studies-downregulation of ELAVL3 and upregulation of HNRPA1 and NOL8. Of these, ELAVL3 was the most significantly downregulated in magnitude, statistical significance, and reproducibility (Fig. 1) -in sALS, its expression was reduced to $42 \%$ of normal ( $\log 2$ fold change -1.2581 with $p=0.0009)$ [48] or $44 \%$ of normal ( $\log 2$ fold change -1.1831 with $p=4.00 \mathrm{E}-08$ ) [32] (Fig. 1a, b). The other RBPs that were differentially expressed in both data sets were both slightly upregulated: hnRNPA 1 ( $\log 2$ fold change $1.22, p=0.02$ [48] and $\log 2$ fold change 1.01396, $p=0.016$ [32]) and NOL8 $(\log 2$ fold change $0.20, p=0.055$ [48] and $\log 2$ fold change $0.56, p=0.001$ [32]) (Supplementary Table 3). TARDBP transcript expression differences indicated possible slight upregulation, not downregulation, in ALS (Fig. 1c), suggesting a compensatory TARDBP autoregulation. Interestingly, other ALS candidate RBPs including FUS, TAF15, hnRNPA2B1, EWS, and MATR3 were not consistently differentially expressed. We validated the RNA levels of ELAVL3 and TARDBP in frozen spinal cord tissues different from those tissues used in our published reports using qPCR ( 3 control and 7 sALS) and again found significant downregulation of ELAVL3 ( $\log 2$ fold change $0.04, p=0.007)$ and not TARDBP ( $\log 2$ fold change $0.34, p=0.80$ ) (Fig. 1d, e). For one of the nervous systems, two different regions of spinal cord (high thoracic and low lumbar) were tested to see if different amounts and subtypes of alpha motor neurons at different spinal cord level were reproducible and no obvious intra- or inter-sample differences of the downregulation of ELAVL3 RNA levels were noted (data not shown). These supported that our findings in the laser capture microdissection studies are reproducible and consistent.

\section{ELAVL3 mislocalizes from the nucleus}

We studied ELAVL3 neuropathologically using IHC and IF (Fig. 1 and Supplementary Fig. 1). As predicted from the transcriptome expression data, in sALS, there were striking abnormalities of ELAVL3 protein expression in alpha motor neurons (Fig. 1f, g and Fig. 2). In control nervous systems, ELAVL3 was clearly observed in nuclei and diffusely in the cytoplasm of essentially all spinal alpha motor neurons (Figs. 1f, 2a-d, 3a). ELAVL3 was exclusively neuronal, and we did not observe nuclear or cytoplasmic presence in glial cells. In sALS, abnormalities had two main neuropathological phenotypes: depletion of normal nuclear ELAVL3 (Figs. 1g, 2e-h) and cytoplasmic accumulations, mainly as dot-like inclusions and only rarely as thread-like or fibrillarlike skeins (Figs. 2f, 3h, i, respectively). ELAVL3 was also observed as diffuse staining along axons and dendrites (Supplementary Fig. 2). While the previous studies in a mouse model of ELAVL3 KO showed spheroid formations along the axons in Purkinje cells in cerebellum [22], we did not observe such formations in axons in anterior horns of sALS patients using MAP2 and TUJ1 markers (Suppl. Fig. 2). ELAVL3 often accumulated in the nucleolus of neurons in which there was ELAVL3 nuclear depletion, interesting in light of our recently reported observation of nucleolar abnormalities in sALS (Supplementary Fig. 3) [1]. We also observed nuclear ELAVL3 depletion from nuclei and rare dot-like aggregates in neurons located in layer $\mathrm{V}$ of motor cortex of sALS (Supplementary Fig. 4). Interestingly, in one control nervous system from an individual who carried an ATXN2 intermediate length repeat expansion but did not have neurological disease, we observed rare nuclear depletion of ELAVL3 and dot-like inclusions in a few neurons in the motor cortex but not spinal cord (Supplementary Fig. 5). These findings establish that ELAVL3 is a significant RBP in sALS neuropathology. 
a

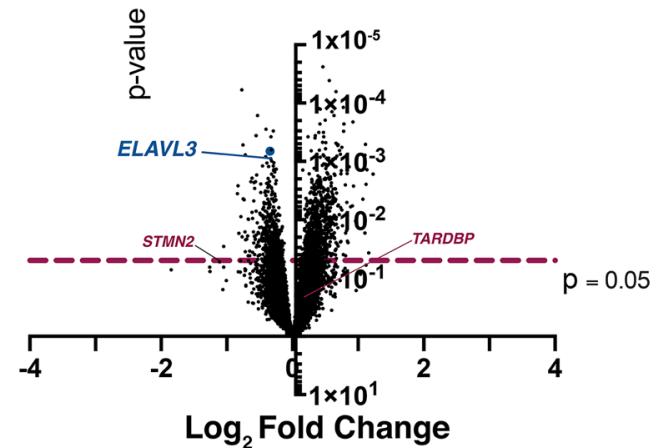

b

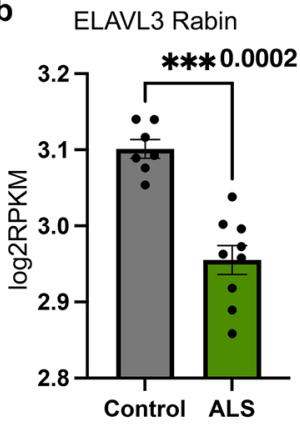

ELAVL3 Krach

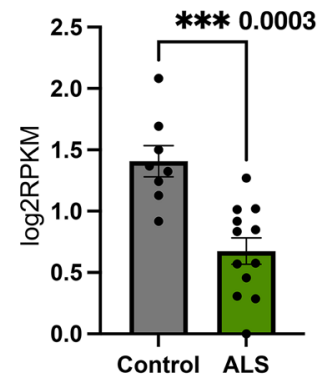

d

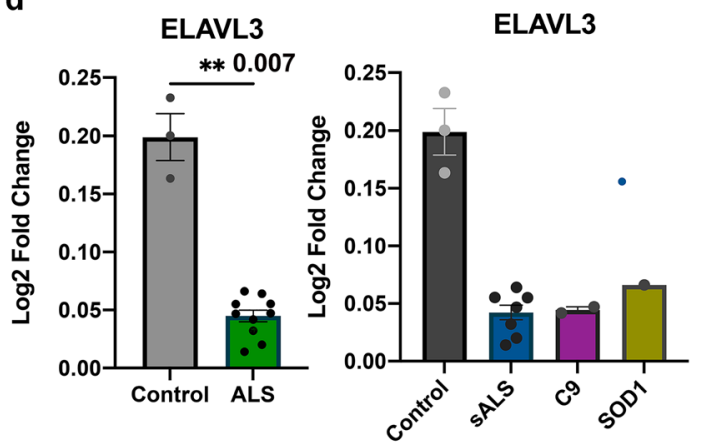

DAPI ELAVL3

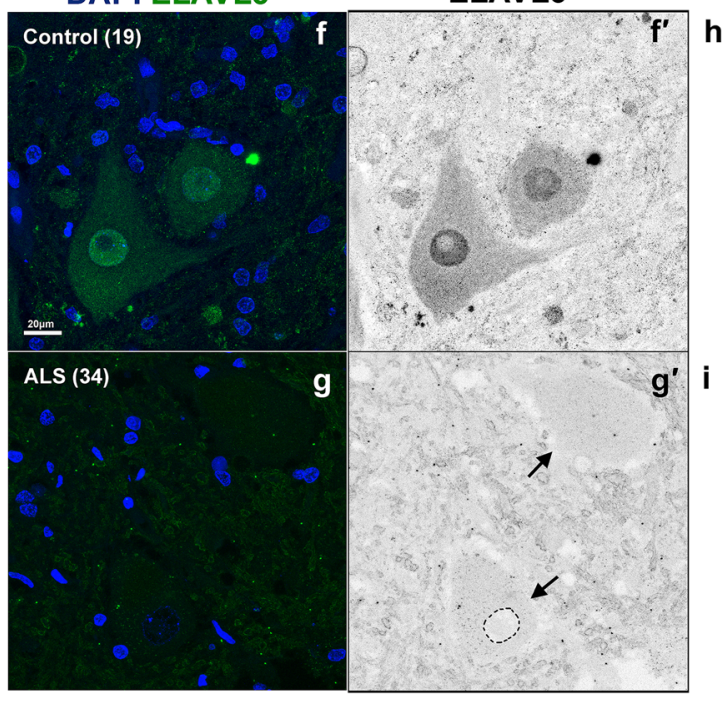

Volcano plot LCM (Krach et al 2018)
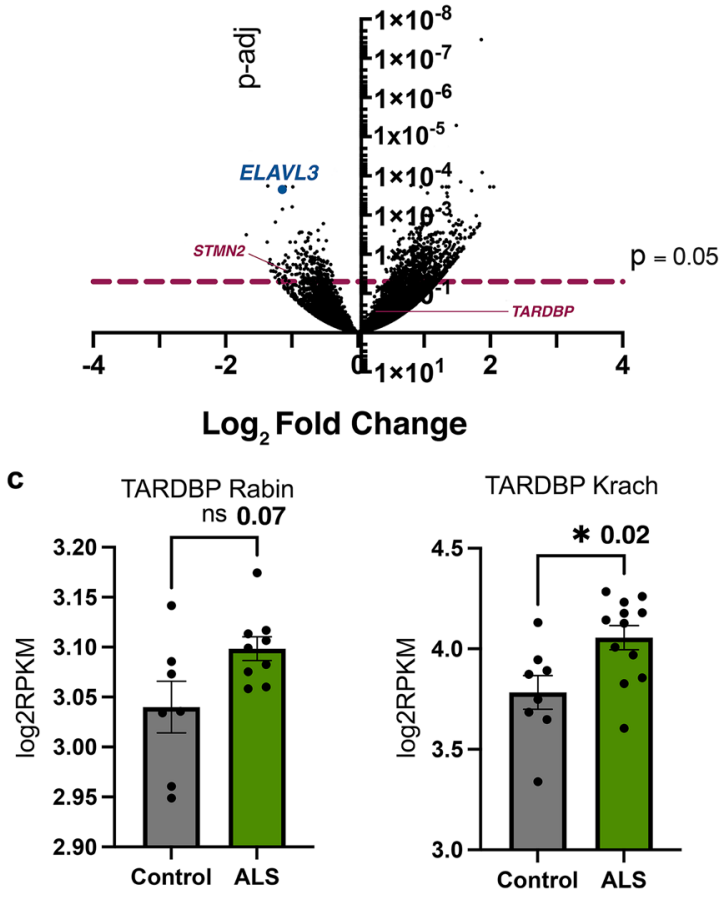

e

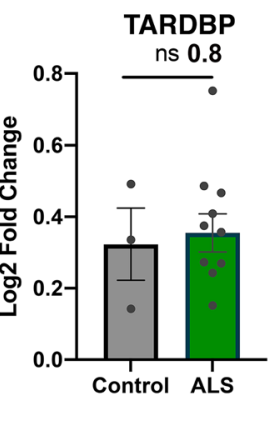

TARDBP
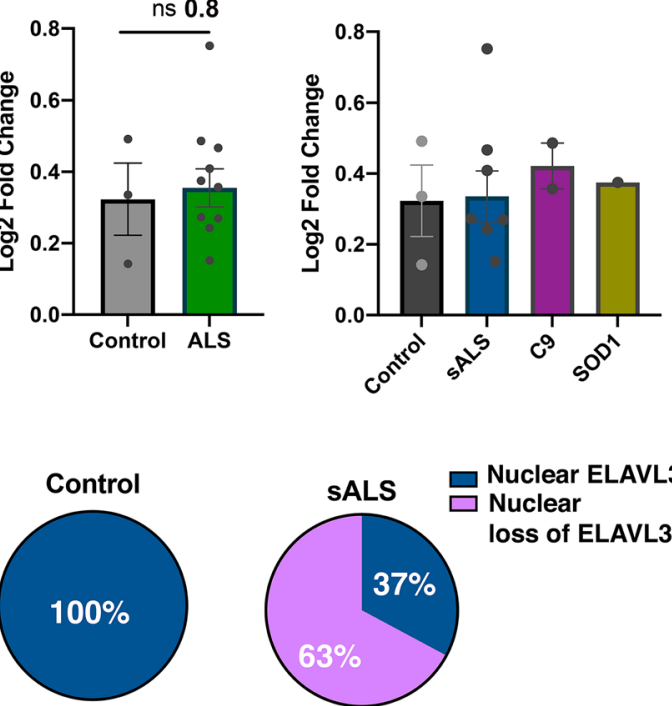

80 neurons 5 patients
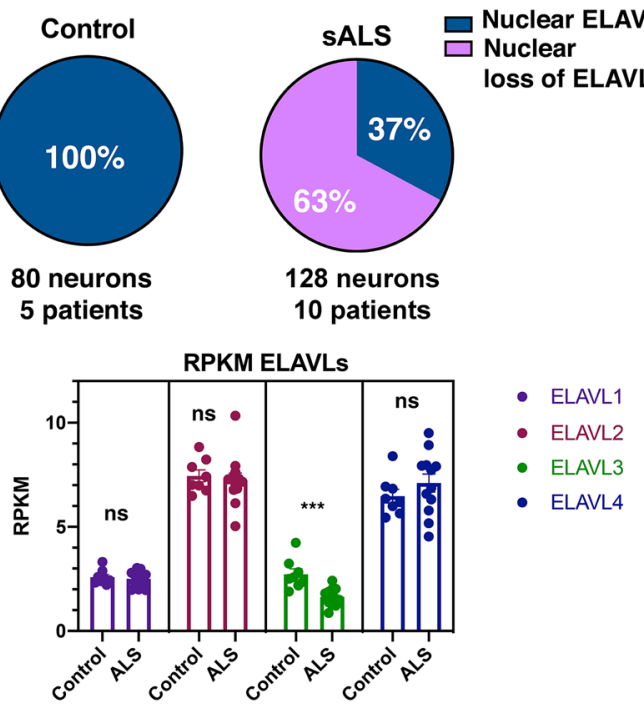
4Fig. 1 ELAVL3 downregulation in sALS motor neurons. a Volcano plots of RNAs from laser captured motor neurons comparing sALS and control showing significant ELAVL3 downregulation in two independent studies. Raw expression values for ELAVL3 (b) and TARDBP (c). Validation by qPCR of bulk tissue (left) and by ALS subcategories (right) of $E L A V L 3$ (d) and TARDBP (e). Immunofluorescence of motor neurons of human spinal cords in control (f) and sALS $(\mathbf{g})$. $\mathbf{h}$ Pie chart of frequency of ELAVL3 nuclear location and depletion. $\mathbf{i}$ Expression of other ELAVLs family members. Scale bar $20 \mu \mathrm{m}$

\section{Nuclear depletion of ELAVL3 rather than cytoplasmic aggregation is characteristic}

We quantified ELAVL3 abnormalities in 161 motor neurons from 11 sALS nervous systems in the spinal cord using IF. In this, we included only motor neurons that had observable nuclei as identified by DAPI staining (Table 1). The average number of such motor neurons was 15 in each nervous system and the range was 6-35. Overall, 114 (71\%) of the motor neurons examined had depletion of nuclear ELAVL3 and 48 (42\%) had cytoplasmic accumulations of ELAVL3 (Fig. 1h and Table 1). Of the 114 motor neurons that had nuclear depletion, 48 (42\%) also had cytoplasmic accumulations of ELAVL3 and 66 (58\%) did not. Of the 48 motor neurons that had cytoplasmic accumulations of ELAVL3, all 48 had nuclear depletion. These findings suggest that ELAVL3 nuclear depletion is more significant than cytoplasmic accumulations in sALS $(p<0.00001$ using Chi-square analysis and Supplementary Table 4). To evaluate ubiquitination, we co-stained those tissues in which ELAVL3 cytoplasmic inclusions were visualized and we did not find colocalization between ELAVL3 and ubiquitin-in these, we did indeed observe ubiquitinated cytoplasmic inclusions, consistent with ubiquitination of TDP-43 (Supplementary Fig. 5).

\section{ELAVL3 abnormalities are also seen in fALS nervous systems including SOD1 mutant ALS}

We compared ELAVL3 in nervous systems with known genetic contributions. $85 \%$ of 59 motor neurons examined in two sALS nervous systems with intermediate length repeat expansions of ATXN2 (Fig. 3b, c) and $40 \%$ of 77 motor neurons examined in five C9ORF72 fALS had depletion of nuclear ELAVL3 (Fig. 3d-f). While it is well recognized that nuclear depletion and cytoplasmic aggregation of TDP43 do not occur in SOD1 ALS [37], surprisingly we found that $48 \%$ of 33 residual motor neurons examined in four SOD1 ALS nervous systems also had nuclear depletion of ELAVL3 (Fig. 3g-i). Additionally, we found ELAVL3 but not TARDBP RNA levels appeared to be down in frozen spinal cord tissues from 2 C9orf72 and 1 SOD1 nervous systems (Fig. 1d). These results support our observation of depleted ELAVL3 in familial cases in tissue samples.
Relevant to this, we looked at ELAVL3 in three ALSrelated mouse models-TDP-43 ${ }^{\triangle \mathrm{NLS}}$, C9orf72, and SOD1 (G93A) - and our preliminary observations in three mice of each model and three respective wild-type littermates support the observation of ELAVL3 nuclear depletion and align with our observations in human samples, although additional studies are needed (Supplementary Fig. 6).

\section{Reduced ELAVL3 and short isoform is present in SALS and fALS}

To confirm the downregulation of ELAVL3 observed transcriptionally and by immunofluorescence, we measured ELAVL3 protein levels by immunoblotting in sALS, C9, and SOD1 ALS cases (Fig. 4a). Control samples had a prominent band of ELAVL3 at $~ 39 \mathrm{kDa}$ corresponding to ELAVL3 full-length protein. In every ALS case, we observed a consistent clear depletion of full-length ELAVL3. Strikingly, a lower band of $\sim 36 \mathrm{kDa}$ appeared in SALS and fALS cases, the appearance of which was associated with the decrease of $39 \mathrm{kDa}$ band, but at $\sim 1 / 3$ the abundance seen in non-ALS tissue (Fig. 4b). To ensure that ELAVL3 depletion was not due to neuronal loss in ALS tissue, we also measured neuronal marker TUJ1 and confirmed that the decrease of ELAVL3 at protein level was independent of lack of neurons in the tissues. This shorter form of ELAV3 is calculated to be 25-30 amino acids shorter than the full-length form. Knowing that a cryptic exon in intron 3 of ELAVL3 was identified by knocking down TDP-43 in a cellular model [31], we sequenced from exon 3 to exon 4 in sALS and control spinal cord tissue samples and did not find either a cryptic exon or a difference (data not shown). In addition, we interrogated RNA-seq expression data and did not find differences [32, 39] (Supplementary Fig. 7). For interest, other differentially expressed genes in ALS whose misregulation could be related to either TDP-43 or ELAVL3 mRNA binding are shown in Supplementary Tables 5-8. To determine if this shorter isoform might be related to post-translational cleavage such as at caspase and calpain cleavage, we interrogated known databases and protein cleavage tools and did not identify an obvious site that would result in 25-30 amino acid shortage. Thus, the exact nature of the short isoform is unclear, and it might be a product of degradation or dephosphorylation.

\section{ELAVL3 abnormalities are more prevalent and earlier than TDP-43 abnormalities}

We evaluated TDP-43 in the same 161 motor neurons from the 11 sALS nervous systems using co-IF. TDP43 was well visualized in the nuclei of neurons and glia cells in control tissue, and sALS patients' tissue exhibited the expected mix of TDP-43 nuclear depletion and 


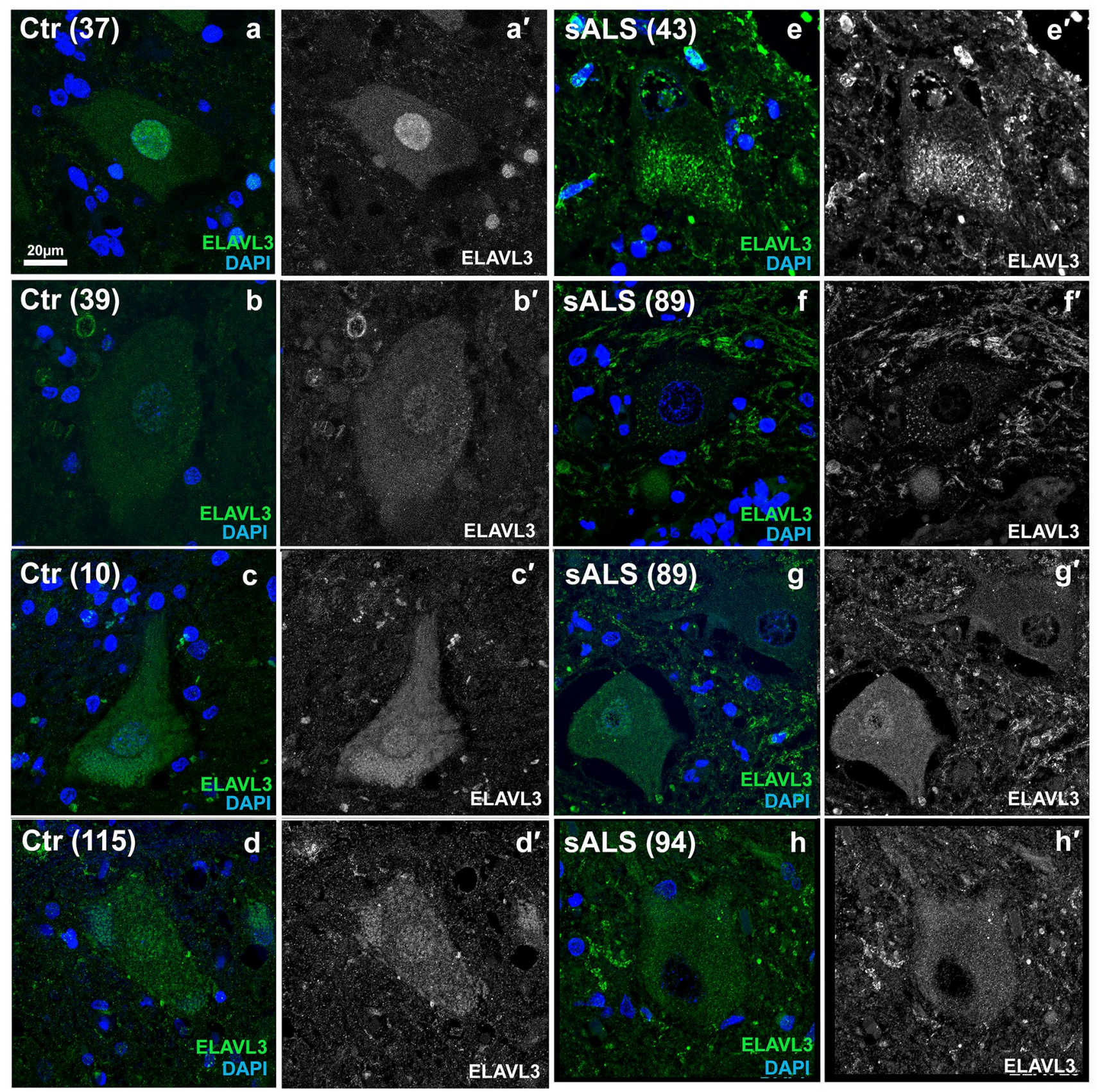

Fig. 2 Immunofluorescence of spinal motor neurons in sALS. a-d Control motor neurons showing ELAVL3 are nuclear and slightly cytoplasmic. e-h SALS motor neurons showing ELAVL3 depleted in

the nucleus, decreased in cytoplasm, and rare cytoplasmic inclusions (f). ELAVL3 green, DAPI blue, scale bar $20 \mu \mathrm{m}$

cytoplasmic accumulations, especially aggregates (Table 1 and Supplementary Fig. 8). Overall, 74 (46\%) of the motor neurons had depletion of nuclear TDP-43 and 59 (37\%) had cytoplasmic accumulations of TDP-43. Of the 74 motor neurons that had depletion of nuclear TDP-43, $54(73 \%)$ also had cytoplasmic accumulations of TDP43 and $20(27 \%)$ did not. Of the 59 motor neurons that had cytoplasmic accumulations of TDP-43, 54 (92\%) also

had nuclear depletion. Nuclear depletion and cytoplasmic aggregates were not significantly different $(p=0.09$ using Chi-square analysis and Supplementary Table 4). This supports current consensus that TDP-43 sALS neuropathology has both nuclear depletion and cytoplasmic accumulation and contrasts to ELAVL3, where nuclear depletion is the hallmark neuropathologic change. 

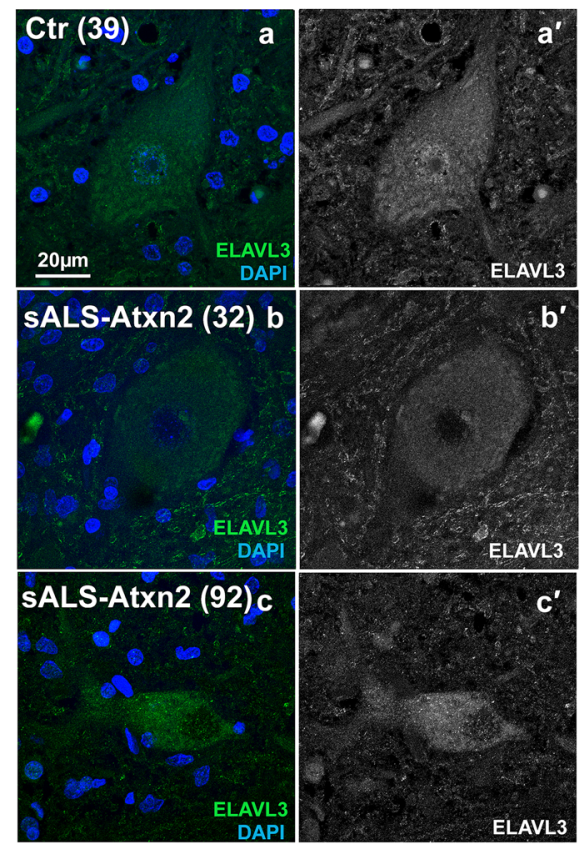

c'
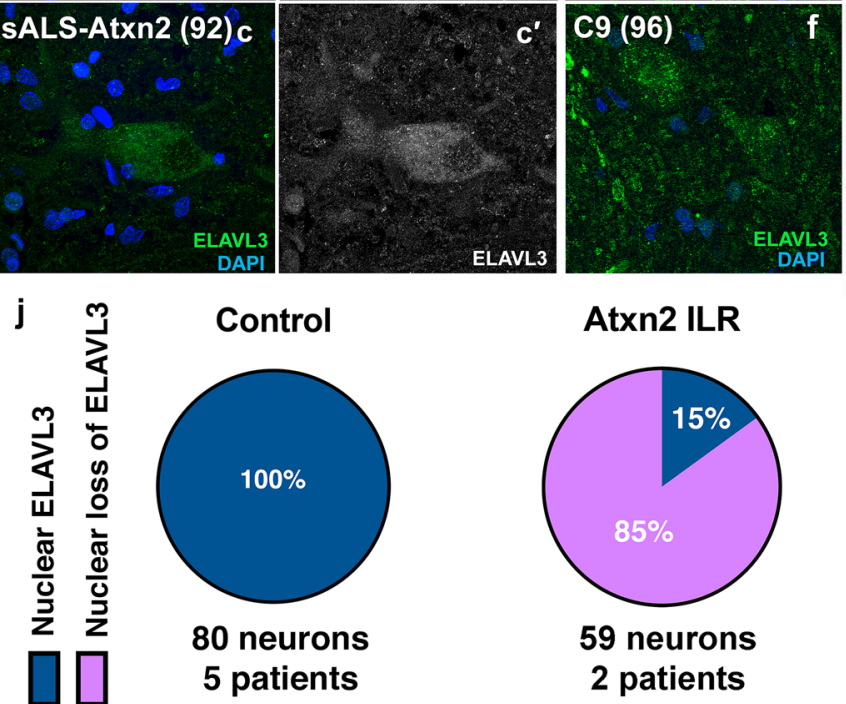

Fig. 3 Immunofluorescence of spinal motor neurons in fALS. a Control motor neurons show nuclear and cytoplasmic ELAVL3. b, c SALS with intermediate length expansions in ATXN2 showing nuclear depletion and cytoplasmic inclusions. d, f C9orf72 ALS motor neurons showing nuclear depletion and occasional fibrillary-

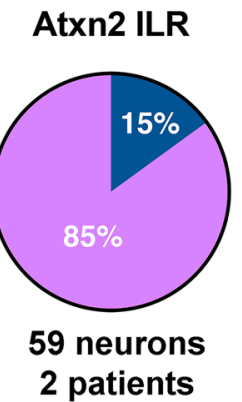

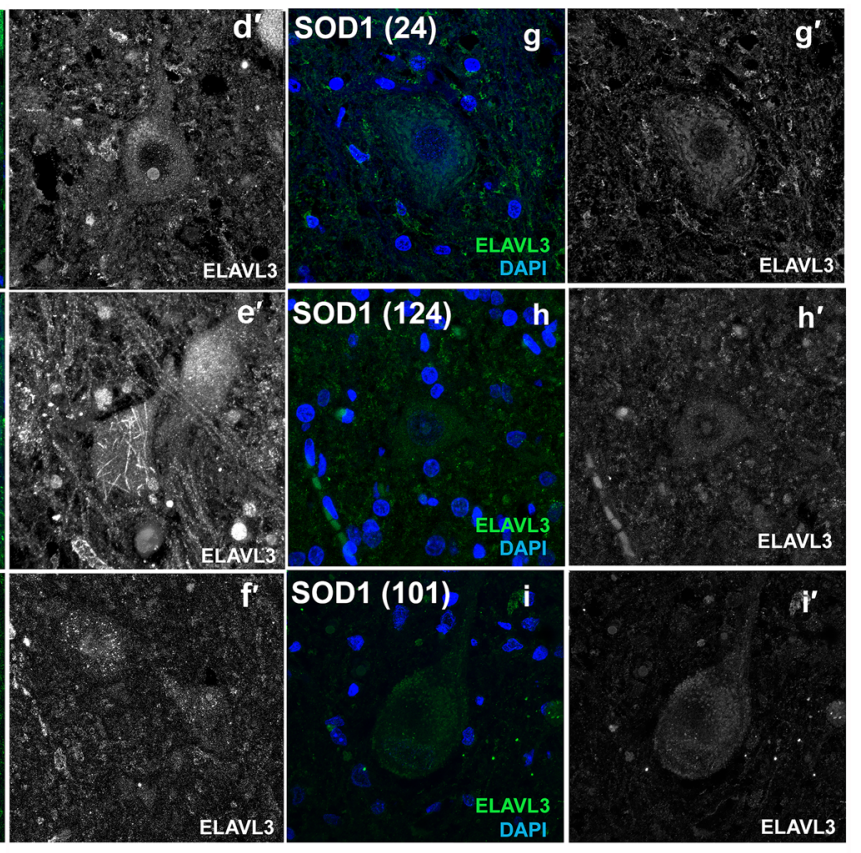

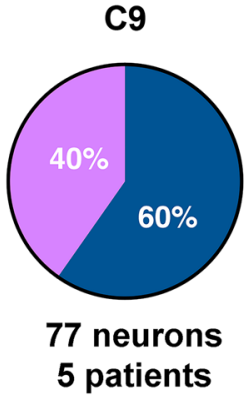

SOD1

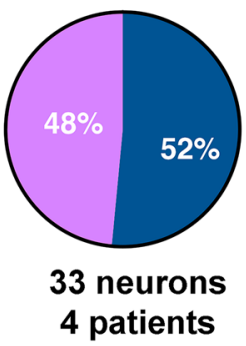

like (e) and dot-like inclusions (f) are seen. g-i SOD1 ALS motor neurons showing nuclear depletion and dot-like inclusion (i). j Pie chart of frequency of ELAVL3 nuclear location and depletion in fALS. ELAVL3 green, DAPI blue, scale bar $20 \mu \mathrm{m}$
Table 1 sALS key pathological findings ( $n=11$ nervous systems, 161 neurons)

\begin{tabular}{lcc}
\hline & ELAVL3 & TDP-43 \\
\hline Neurons with nuclear depletion, total & $114(71 \%)$ & $74(46 \%)$ \\
•Neurons with nuclear depletion that have cytoplasmic inclusions & $48(42 \%)$ & $54(73 \%)$ \\
•Neurons with nuclear depletion that do not have cytoplasmic inclusions & $66(58 \%)$ & $20(27 \%)$ \\
Neurons with cytoplasmic inclusions, total & $48(30 \%)$ & $59(37 \%)$ \\
$\bullet$ Neurons with cytoplasmic inclusions that also have nuclear depletion & $48(100 \%)$ & $54(92 \%)$ \\
$\bullet$ Neurons with cytoplasmic inclusions that do not nuclear depletion & $0(0 \%)$ & $5(8 \%)$ \\
\hline
\end{tabular}

We then directly compared ELAVL3 and TDP-43 abnormalities in the same neurons (Fig. 5a-d). Neurons with nuclear depletion of ELAVL3 were more frequent than TDP-43 abnormalities and encompassed all TDP-43 pathology (Supplementary Table 4). $46 \%$ of neurons with nuclear depletion of ELAVL3 had abnormal TDP-43 (either nuclear depletion or cytoplasmic accumulation), but $100 \%$ of neurons with pathological TDP-43 also had nuclear depletion of ELAVL3 (Fig. 5e-h). In addition, we found almost all cytoplasmic pathological inclusions did not colocalize (Supplementary Fig. 9). We compared ELAVL3 and TDP43 in the fALS nervous systems with ATXN2 intermediate 
Fig. 4 Immunoblotting of spinal cord in sALS and fALS. a Western blot of healthy donors, sALS and fALS spinal cords for neuronal marker TUJ1, TDP43, ELAVL3, and GAPDH. b Quantifications of ELAVL3 and TDP-43 normalized to GAPDH in western blot

a
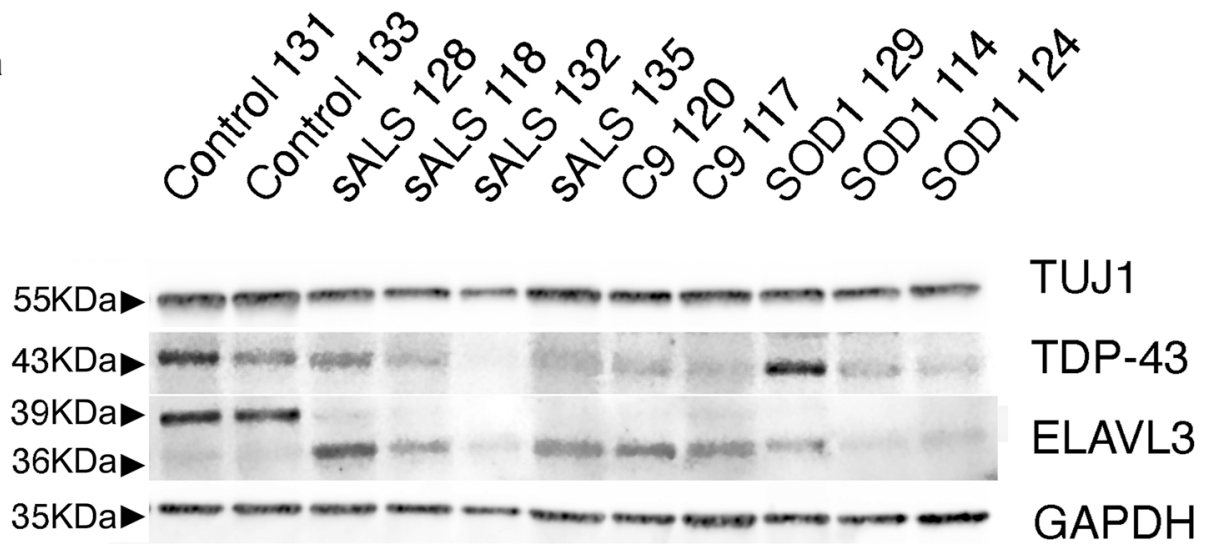

b

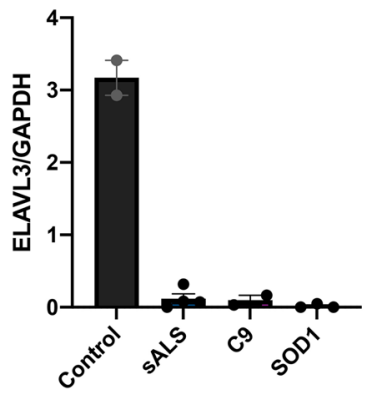

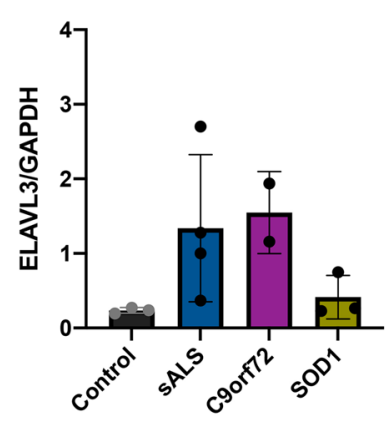

ELAVL3 short isoform

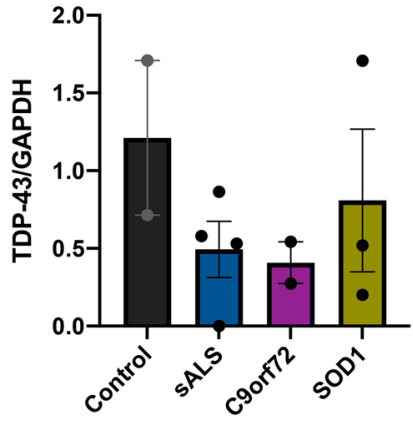

TDP-43 expansions and C9ORF72 and observed depletion of nuclear ELAVL3 even when TDP-43 was nuclear (Fig. 5e-h). Importantly, in nervous systems from SOD1 mutations, we again observed ELAVL3 abnormalities (33\% of neurons) and did not observe TDP-43 pathology in ELAVL3-abnormal neurons, where TDP-43 was indeed nuclear (Fig. 5e-h).

\section{ELAVL3 nuclear depletion is upstream of TDP-43 in a cellular model}

Since our results suggest that ELAVL3 abnormalities may be upstream of TDP-43 abnormalities, we used a neuron-like cellular model under prolonged stress to study the temporal dynamics including nuclear depletion, cytoplasmic localization, and inclusion formation of both proteins. We applied Rapamycin, a common ER stressor, to cell cycle-arrested SH-SY5Y cells which had endogenous TDP-43 tagged with GFP [15]. One day after the stress was applied, ELAVL3 was mislocalized and formed cytoplasmic inclusions, while TDP-43 remained predominantly nuclear (Supplementary Fig. 10). Co-staining ELAVL3 with stress granule marker G3BP showed variable colocalization of ELAVL3 with G3BP as well as TDP-43 with G3BP. TDP-43 remained normal until 4 weeks, at which time it was depleted in the nucleus and formed dot-like inclusions in the cytoplasm.
This model supports that ELAVL3 abnormalities occur earlier and are upstream of TDP-43 abnormalities.

\section{Other ELAVL family members show variable and infrequent pathology}

Because ELAVLs family members have high sequence homology and may have redundant functions in the nervous system, we analyzed the expression pattern of ELAVL1 $(\mathrm{Ctr}=2$ and sALS $=6)$, ELAVL2 $(\mathrm{Ctr}=4$ and $\operatorname{sALS}=10)$, and ELAVL4 $(\mathrm{Ctr}=6$ and $\mathrm{sALS}=31)$. ELAVL1 was expressed in the nuclei and diffusely in the cytoplasm of neurons and glial cells in control nervous systems (Fig. 6). In sALS, C9, and SOD1 nervous systems, nuclear expression of ELAVL1 was usually similar to controls but more variable expressed, and rare cytoplasmic inclusions were seen, occasionally colocalizing with TDP-43 speckles or encapsulating TDP-43 aggregates in the cytoplasm (Fig. 6a-e and Supplementary Fig. 11). ELAVL2 was normally expressed in the nuclei and diffusely in the cytoplasm of neurons but not glial cells in control nervous systems. In sALS and C9 nervous systems, ELAVL2 was variably reduced in the nucleus and colocalized with fibrillar aggregates of TDP-43 in the cytoplasm (Fig. 6f, j). We did not observe ELAVL2 abnormalities in SOD1 nervous systems. ELAVL4 was 


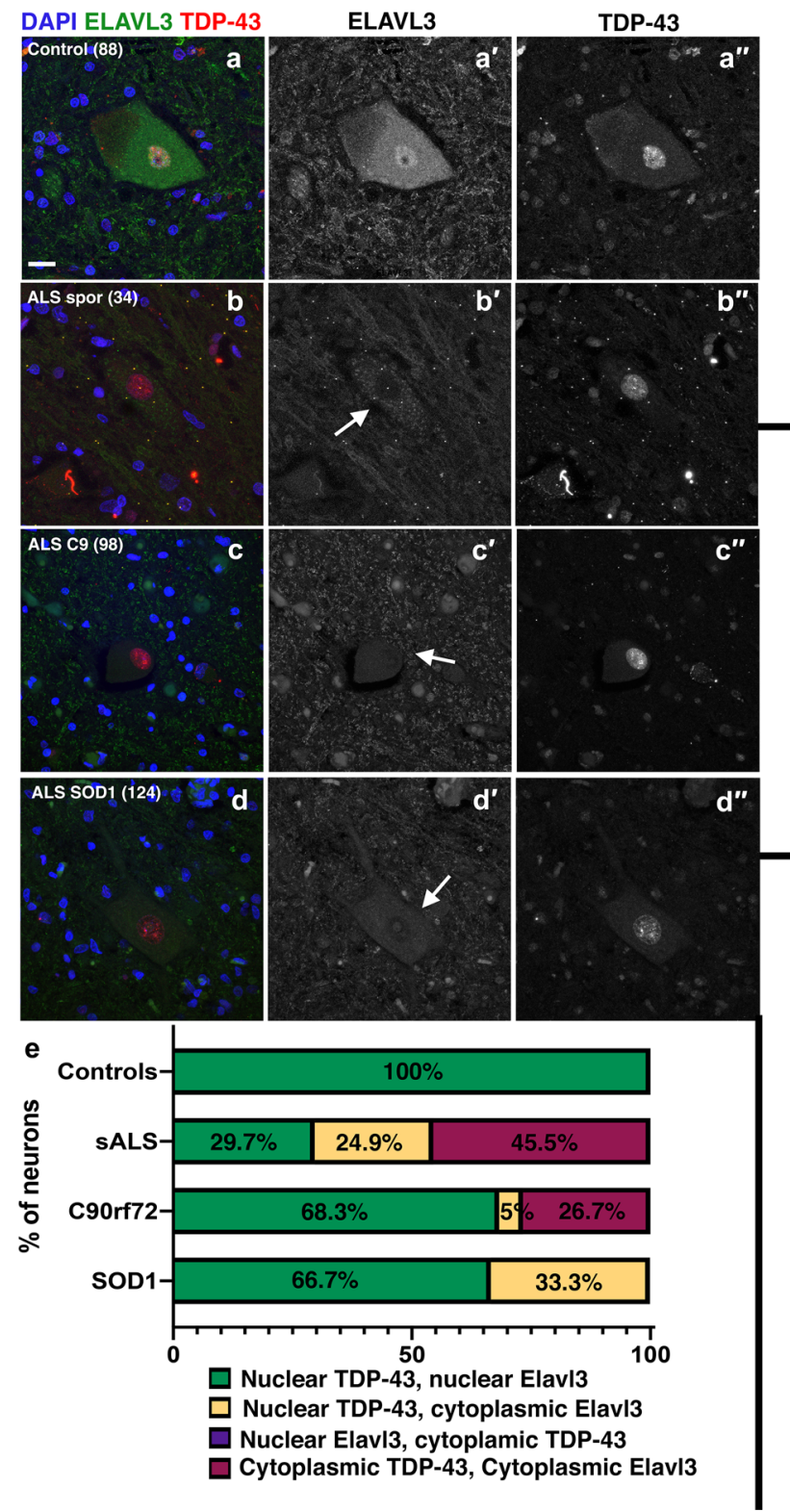

Fig. 5 ELAVL3 abnormalities are upstream of TDP-43. Doublelabeled immunofluorescence of ELAVL3 and TDP-43 in spinal motor neurons. a Control motor neuron showing ELAVL3 is present in the nucleus and diffuse in the cytoplasm and TDP-43 is nuclear. Motor neurons from sALS (b), C9orf72 (c), and SOD1 (d) show ELAVL3 nuclear depletion is observed when TDP-43 is nuclear (b-d). e Statis-

variably expressed in the nucleus and cytoplasm of neurons but not glia in controls. In sALS, C9, and SOD1 nervous systems, nuclear expression of ELAVL4 was more variable expressed than controls, and cytoplasmic inclusions were seen usually as dot-like inclusions occasionally colocalizing with TDP-43 (Fig. 6k-o and Supplementary Fig. 11). Since we have seen other candidate proteins variably colocalize with TDP-43 by immunofluorescence (data not shown), the neuropathologic and biological specificity
Nuclear ELAVL3

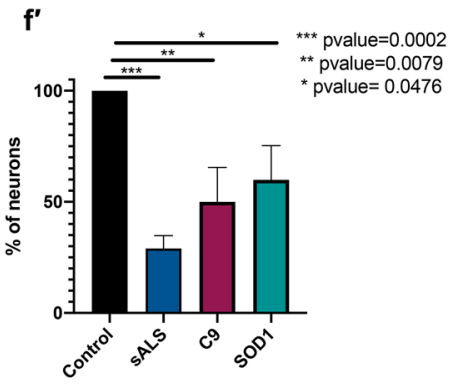

f

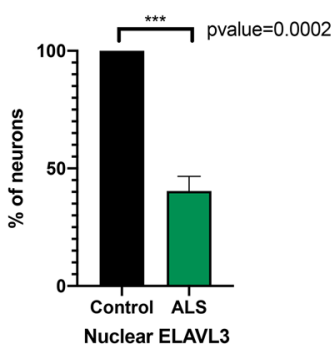

Nuclear TDP-43

g ${ }^{* *}$ pvalue $=0.0019$

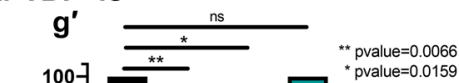

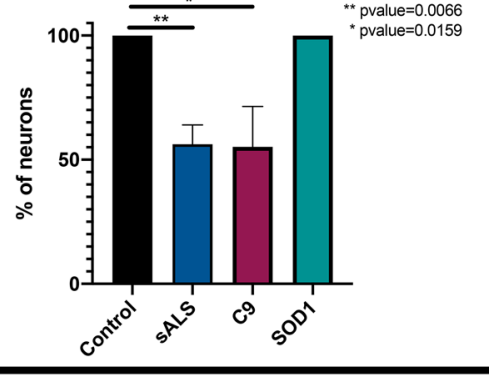

h
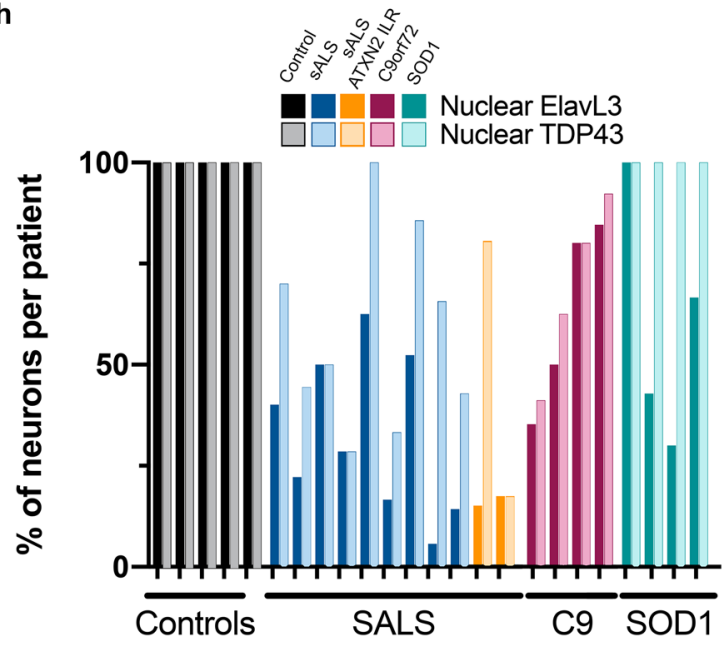

tical comparison of ELAVL3 and TDP-43 abnormalities in the same neurons. f Statistical analysis of ELAVL3 abnormalities in ALS overall and in sorted groups. $\mathrm{g}$ Statistical analysis of TDP-43 abnormalities in ALS overall and in sorted groups. $\mathbf{h}$ Comparison and variance of ELAVL3 and TDP-43 abnormalities in each nervous system sorted by groups. ELAVL3 green, TDP-43 red, DAPI blue, scale bar $20 \mu \mathrm{m}$

and significance and possible interactions with TDP-43. To compare all four proteins to each other and establish variability in the same nervous systems, three nervous systems were examined for all four ELAVLs $(\mathrm{Ctr}=1$, $\operatorname{sALS}=1$ and SOD $1=1$ ). We found that only ELAVL3 was consistently and significantly abnormal (Table 2 and Supplementary Fig. 11). Taken together, these findings suggest that ELAVL3 is unique within the ELAVL family. 

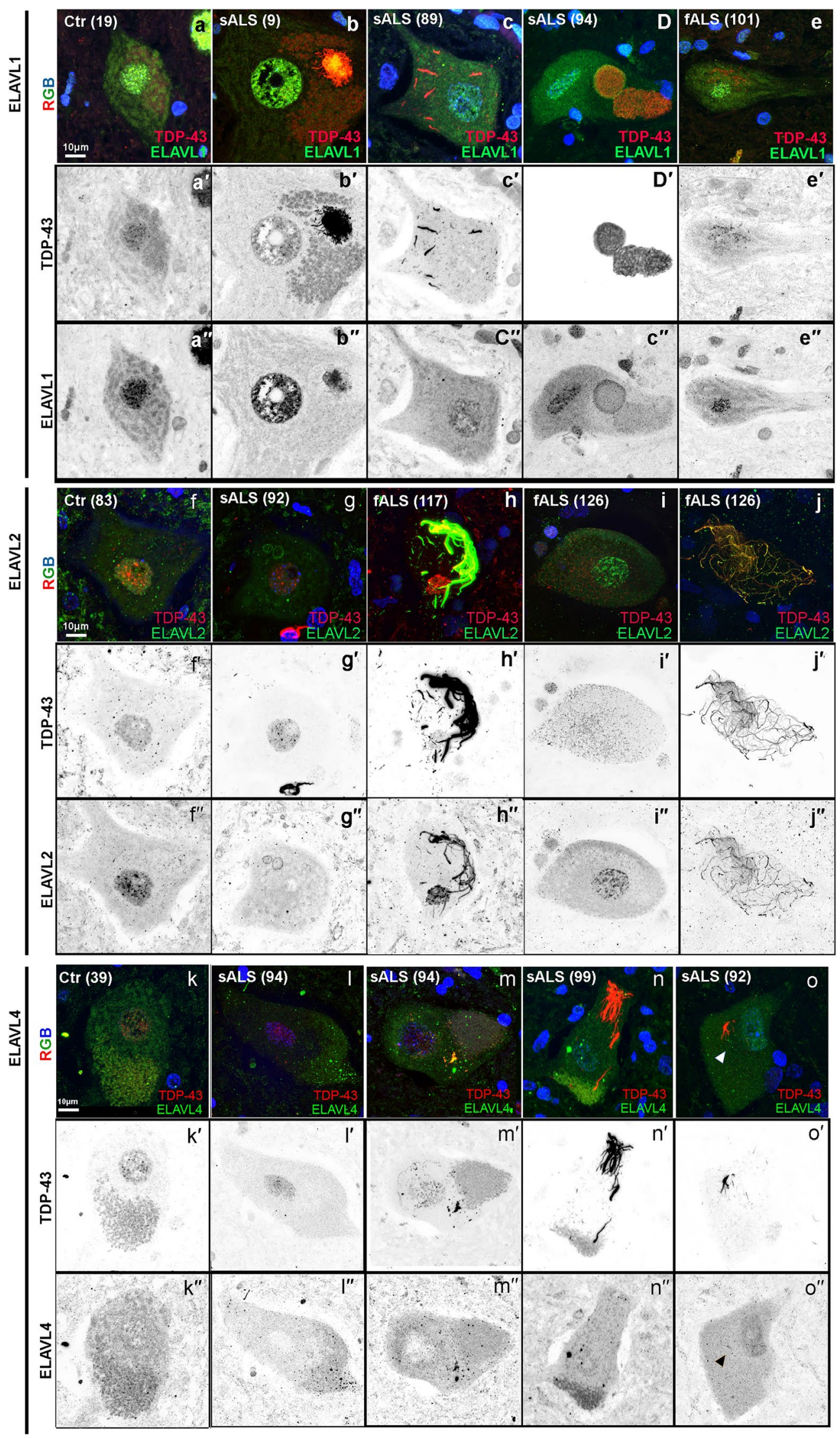
४Fig. 6 ELAVL family members are also frequently abnormal but only in the presence of TDP-43 aggregates. ELAVL1 in control (a), sporadic (b-d), and SOD1 (e). Nuclear ELAVL1 expression is maintained or slightly reduced and, in the cytoplasm, can be seen either colocalizing or encapsulating TDP-43 aggregates or forming its own inclusions. ELAVL2 expression in control (f), sporadic (g), and C9orf72 (h-j). Nuclear ELAVL2 expression is maintained or reduce depending on the ALS sample and in the cytoplasm can be seen either colocalizing TDP-43 fibrillary aggregates $(\mathbf{h}-\mathbf{j})$, but not with speckles (i). ELAVL4 in control (k-l) and sporadic samples $(\mathbf{m}-\mathbf{0})$. Nuclear ELAVL4 expression is more diffuse in controls than other ELAVLS, and in ALS, it is reduced or depleted from the nucleus and it variably colocalizes with TDP-43 aggregates in the cytoplasm

\section{There are no ELAVL genetic variants associated with sporadic ALS}

To investigate the contribution of common ELAVL variants to disease risk, we performed association testing on 750 common variants present in ELAVL1,2,3, and 4 genes in 10,599 cases and 42,279 controls [43]. There were no variants within these genes that passed the genome-wide threshold of $5 \times 10^{-8}$, implying that these common variants do not have a significant effect on risk at the population level in sALS cases (Supplementary Fig. 12). At the rare variant level, we performed a gene-based analysis on variants that were predicted to be missense or have a loss-of-function effect using the SKAT-O method and did not find excess presence of rare variants in cases compared to controls. From these results, it does not appear to be that there are rare variants in these genes that drive/modify risk for ALS; at least this is true for this set of imputed variants in the tested cohort.

\section{Discussion}

Because of the focal clinical onset of ALS and anatomical propagation of disease over time, the timing of degeneration desynchronizes in motor neurons, and they display different degrees of molecular changes neuropathologically [49]. This allowed us to identify upstream candidates using a genomics approach $[32,48]$, and from this, we identified ELAVL3 as a candidate RBP of interest. Indeed, we found striking neuropathological abnormalities of ELAVL3 in motor neurons of sporadic and familial ALS tissue samples. The abnormalities were of two varieties-nuclear depletion and cytoplasmic accumulation - and of these, nuclear depletion was hallmark. The importance of nuclear depletion is further supported by the fact that ELAVL3 had reduced expression at both the RNA and the protein levels. Thus, the evidence supports that nuclear depletion and loss of function rather than subcellular location or cytoplasmic accumulation and toxicity are key to the mechanism by which ELAVL3 may be involved in pathogenesis. The neuropathological differences between cytoplasmic accumulation and nuclear depletion that we observed are unlikely due to sampling, since the cytoplasmic accumulations are diffusely scattered in the cytoplasm and the cross-sectional areas of cytoplasm are significantly greater than cross-sectional areas of nucleus, which would bias in the opposite direction. The difference is also unlikely to be due to the detection threshold of antibody, since it is equal in either subcellular location. However, it is known that abnormalities of the karyopherin nuclear transport protein family members can be both cause and consequence of protein mislocalization and aggregate formation in degenerative proteinopathies and remains to be defined for ELAVL3.

It is not unreasonable to think ELAVL3 pathobiology in ALS may be different from TDP-43. In TDP-43 pathobiology, the preponderance of evidence indicates that subcellular mislocalization rather than expression is key. Both nuclear depletion and cytoplasmic accumulation of TDP-43 usually appear together neuropathologically, and our neuropathological data indicate both nuclear loss and cytoplasmic accumulation are significant. TDP-43 expression is tightly regulated and much data support that it is not differentially expressed although our own transcriptome data if anything suggest slight upregulation. Unlike TDP-43, ELAVL3 does not have a canonical nuclear localization signal (NLS), or a low complexity glycine-rich prion domain and it is significantly downregulated at the RNA and protein levels. Our data further suggest that ELAVL3 abnormalities are upstream of TDP-43 in the process of motor neuron degeneration. This is supported by three lines of reasoning. First, all neurons with TDP-43 abnormalities displayed ELAVL3 abnormalities, but many neurons with ELAVL3 abnormalities displayed normal nuclear TDP-43. Thus, TDP-43 pathology was a subset of ELAVL3 pathology, not vice versa. Second, ELAVL3 abnormalities were also seen in SOD1 nervous systems, where TDP-43 abnormalities are not present. The role SOD1 plays in sALS is debated. Regardless, it is accurate to say that ELAVL3 pathology is widespread and prevalent in sALS and fALS from the two most common mutations SOD1 and C9orf72. Neither SOD1 nor TDP-43 are as clearly generalizable neuropathologically as ELAVL3 is, although it is possible that TDP-43 or SOD1 are abnormal at a molecular level that are not visible neuropathologically. Third, our cell culture data in which cell cycle arrested nondividing neuron-like TDP-43-GFP SH-SY5Y cells were artificially stressed clearly showed ELAVL3 abnormalities long before TDP-43 abnormalities, suggesting a more sensitive and prompter response to stress. These results were recently shown in detail in an experimental study reporting uniform downregulation of ELAVL3 in extensive ALS iPSC-MNs models at both RNA and protein levels and, importantly, this decrease was present beginning in early development, suggesting that ELAVL3 is an early modulator of downstream 
Table 2 Summary of ELAVL family

\begin{tabular}{lllll}
\hline $\begin{array}{l}\text { In presence of } \\
\text { TDP-43 pathol- } \\
\text { ogy }\end{array}$ & ELAVL1 & ELAVL2 & ELAVL3 & ELAVL4 \\
\hline NUCLEUS & No change/decrease & No change/decrease & Nuclear loss & No change/decrease \\
CYTOPLASM & No change/decrease & No change/decrease & No change/decrease & No change/decrease \\
& Rare dot-like inclusions & No dot-like inclusions & Dot-like inclusions & Dot-like inclusions \\
& $\begin{array}{l}\text { Colocalization with TDP-43 } \\
\text { or encapsulation of aggre- } \\
\text { gates }\end{array}$ & $\begin{array}{l}\text { Often colocalize with TDP-43 } \\
\text { fibrils but not dot-like inclu- } \\
\text { sions }\end{array}$ & $\begin{array}{l}\text { No colocalization with TDP43. Rare } \\
\text { colocalization with large TDP-43 } \\
\text { aggregates }\end{array}$ & $\begin{array}{c}\text { Sometimes colocal- } \\
\text { ize with TDP-43 } \\
\text { aggregates }\end{array}$ \\
\hline
\end{tabular}

disease [21]. Taken together, these findings are consistent with an upstream effect of ELAVL3 on both SOD1 and TDP-43, an effect that could be explained, for example, by regulation of ARES in the 3'UTR, which both proteins are known to have [3, 12, 41].

The short ELAVL3 isoform was an unexpected finding. Since the changes on immunoblotting mobility are of $3 \mathrm{kDa}$, the abnormal isoform is expected to be 25-30 amino acids shorter than the normal protein. An isoform of ELAVL3 is known to exist, but it is only six amino acids different (isoform 2 is missing amino acids 251-257). Since we used an antibody to exon 2 epitopes near the N-terminal in both the neuropathological and biochemical studies, it seems reasonable to conclude that the shortening is after exon 2. One possibility is inclusion of a cryptic exon from reduction of TDP-43 such as has been identified in STMN2 [31] and UNC13A [6, 50]. Indeed, a cryptic exon due to TDP-43 downregulation has been identified at intron 3 of ELAVL3 [31]. However, we could not identify such in the ALS samples sequencing from exon 3 to exon 4 as well as from mapping our RNA-seq data [32]. This is consistent with our other findings that ELAVL3 abnormalities are independent of TDP-43 mechanisms, recognizing that there is substantial overlap between TDP-43 depletion and ELAVL3 depletion and the known binding of TDP-43 on ELAVL3. The possibility that ELAVL3 protein might undergo cleavage was not supported by caspase or calpain cleavage sites predictions in protein cleavage databases. Thus, at this time, we are unable to further explain the structure and mechanism of the short isoform.

Previous literature has already implicated ELAVL3 in ALS pathogenesis, although it has received little direct attention and is under recognized. In a systematic survey of 213 proteins harboring RNA recognition motifs in a yeast functional screen, ELAVL3 (but not the other ELAVLs) had a high toxicity score and, as stated, did not harbor a prion domain, unlike TDP-43, FUS, and other RBPs [8]. In human neuronal cell model, TDP-43 and FUS depletion both showed changes in ELAVL3, but they were oppositely regulated: downregulated by siTDP-43 and upregulated by siFUS [7]. In a study of TDP-43 loss of function in human motor neurons differentiated from human pluripotent stem cells, ELAVL3 was significantly downregulated, and as mentioned above, a cryptic exon was identified at intron 3 [31]. Also as mentioned above, a recent study of iPSCderived motor neuron models of familial and sporadic ALS found that downregulation of ELAVL3 expression was an early ALS hallmark that began during development and persisted into end stage [21]. Other ELAVLs family members have also been identified in ALS biology and are important in light of our neuropathological characterizations of ELAVL2 and ELAVL4, which showed cytoplasmic accumulation and variable colocalization with TDP-43 aggregates in motor neurons of ALS spinal cords. Since we have observed variable colocalization of TDP-43 and other candidate proteins, the neuropathological and biological specificity and significance remains to be clarified. Other ELAVLs have been shown to be involved as positive gene regulators of SOD1 by binding to ARES in the 3'UTR [41]. ELAVL2 was differentially expressed in motor axons and $\mathrm{NMJ}$, although the findings showed upregulation [51]. In iPSC-derived MN models of FUS mutant ALS, mutant FUS bound ELAVL4 3'UTR, resulting in increased production of the ELAVL4 protein, which were found in cytoplasmic aggregates in the spinal cord of ALS [52]. ELAVL4 has also been shown to be involved in spinal muscular atrophy, another motor neuron disorder [11].

The suggestion that ELAVL3 abnormalities are upstream in the process of neurodegeneration led us to question if genetic factors might be related to its misregulation. However, no significant genetic association of ELAVL3 or other ELAVL family members could be identified, indicating that misregulation of ELAVL3 is likely an early acquired abnormality. While common variant association and rare gene-burden analysis at population scale on sporadic ALS cases do not show significant contribution to risk, ultra-rare variants may exist in families that do not carry the common ALS mutations. In summary, we have compelling evidence implicating ELAVL3 upstream in sporadic, C9orf72, and SOD1 ALS pathogenesis that does not appear to be genetically based. The triggers and upstream regulators and downstream mechanisms in the neurodegeneration cascade remain 
unknown. Further studies will elucidate mechanisms of this important RBP.

Supplementary Information The online version contains supplementary material available at https://doi.org/10.1007/s00401-021-02374-4.

Acknowledgements This research was supported by grants from National Institute of Neurological Disorders and Stroke (NIH R01NS088578 and R21NS121805), UCSD Microscopy Core (NINDS NS047101), ALS Association, Target ALS, Microsoft Research, Pam Golden and the Kraatz Family/Nicholas Martin Jr. Family Foundation. The SNP dataset(s) used for the genetic analyses was obtained from the Age-Related Eye Disease Study (AREDS) Database and provided in the supplements. We thank Target ALS CNS biorepository, the patients, and their families for their generous contribution to this research. We also thank the Nikon Imaging Center at UC San Diego for assistance with microscopy and analysis and UCSD School of Medicine Microscopy Core (Grant P30 NS047101).

Author contributions SDG analyzed the data of previous papers and designed the experiments. MJR did tissue embedding and histology. SDG and MJR performed the staining. VIK did qPCR analysis, imaging, and quantifications. VIK provided mouse tissue and performed staining in mice. OAA helped with cellular models and motor-cortex imaging. SVS did immunoblotting. RC and BT evaluated genetic variants. SDG and JR wrote the paper. SDG, VIK, SVS, OAA, MJR, DWC, BJT, RC, and JR discussed the results and contributed to the final manuscript.

Open Access This article is licensed under a Creative Commons Attribution 4.0 International License, which permits use, sharing, adaptation, distribution and reproduction in any medium or format, as long as you give appropriate credit to the original author(s) and the source, provide a link to the Creative Commons licence, and indicate if changes were made. The images or other third party material in this article are included in the article's Creative Commons licence, unless indicated otherwise in a credit line to the material. If material is not included in the article's Creative Commons licence and your intended use is not permitted by statutory regulation or exceeds the permitted use, you will need to obtain permission directly from the copyright holder. To view a copy of this licence, visit http://creativecommons.org/licenses/by/4.0/.

\section{References}

1. Aladesuyi Arogundade O, Nguyen S, Leung R, Wainio D, Rodriguez M, Ravits J (2021) Nucleolar stress in C9orf72 and sporadic ALS spinal motor neurons precedes TDP-43 mislocalization. Acta Neuropathol Commun 9:26

2. An H, Skelt L, Notaro A, Highley JR, Fox AH, La Bella V et al (2019) ALS-linked FUS mutations confer loss and gain of function in the nucleus by promoting excessive formation of dysfunctional paraspeckles. Acta Neuropathol Commun 7:7

3. Bakheet T, Hitti E, Al-Saif M, Moghrabi WN, Khabar KSA (2018) The AU-rich element landscape across human transcriptome reveals a large proportion in introns and regulation by ELAVL1/ HuR. Biochim Biophys Acta Gene Regul Mech 1861:167-177

4. Barmada SJ, Skibinski G, Korb E, Rao EJ, Wu JY, Finkbeiner S (2010) Cytoplasmic mislocalization of TDP-43 is toxic to neurons and enhanced by a mutation associated with familial amyotrophic lateral sclerosis. J Neurosci 30:639-649
5. Brenner D, Weishaupt JH (2019) Update on amyotrophic lateral sclerosis genetics. Curr Opin Neurol 32:735-739

6. Brown AA-L, Wilkins OG, Keuss MJ, Hill SE, Zanovello M, Lee WC et al (2021) Common ALS/FTD risk variants in UNC13A exacerbate its cryptic splicing 2 and loss upon TDP-43 mislocalization. bioRxiv. https://doi.org/10.1101/2021.04.02.438170

7. Colombrita C, Onesto E, Buratti E, de la Grange P, Gumina V, Baralle FE et al (2015) From transcriptomic to protein level changes in TDP-43 and FUS loss-of-function cell models. Biochim Biophys Acta 1849:1398-1410

8. Couthouis J, Hart MP, Shorter J, DeJesus-Hernandez M, Erion $\mathrm{R}$, Oristano R et al (2011) A yeast functional screen predicts new candidate ALS disease genes. Proc Natl Acad Sci USA 108:20881-20890

9. Cragnaz L, Klima R, Skoko N, Budini M, Feiguin F, Baralle FE (2014) Aggregate formation prevents dTDP-43 neurotoxicity in the Drosophila melanogaster eye. Neurobiol Dis 71:74-80

10. Dalmau J, Furneaux HM, Gralla RJ, Kris MG, Posner JB (1990) Detection of the anti-Hu antibody in the serum of patients with small cell lung cancer-a quantitative western blot analysis. Ann Neurol 27:544-552

11. Fallini C, Zhang H, Su Y, Silani V, Singer RH, Rossoll W et al (2011) The survival of motor neuron (SMN) protein interacts with the mRNA-binding protein $\mathrm{HuD}$ and regulates localization of poly(A) mRNA in primary motor neuron axons. J Neurosci 31:3914-3925

12. Fallmann J, Sedlyarov V, Tanzer A, Kovarik P, Hofacker IL (2016) AREsite2: an enhanced database for the comprehensive investigation of AU/GU/U-rich elements. Nucleic Acids Res 44:D90-D95

13. Fifita JA, Zhang KY, Galper J, Williams KL, McCann EP, Hogan AL et al (2017) Genetic and pathological assessment of hnRNPA1, hnRNPA2/B1, and hnRNPA3 in familial and sporadic amyotrophic lateral sclerosis. Neurodegener Dis 17:304-312

14. Fragkouli A, Koukouraki P, Vlachos IS, Paraskevopoulou MD, Hatzigeorgiou AG, Doxakis E (2017) Neuronal ELAVL proteins utilize AUF-1 as a co-partner to induce neuron-specific alternative splicing of APP. Sci Rep 7:44507

15. Gasset-Rosa F, Lu S, Yu H, Chen C, Melamed Z, Guo L et al (2019) Cytoplasmic TDP-43 de-mixing independent of stress granules drives inhibition of nuclear import, loss of nuclear TDP43, and cell death. Neuron 102:339-357.e7

16. Gilpin KM, Chang L, Monteiro MJ (2015) ALS-linked mutations in ubiquilin-2 or hnRNPA1 reduce interaction between ubiquilin-2 and hnRNPA1. Hum Mol Genet 24:2565-2577

17. Gui X, Luo F, Li Y, Zhou H, Qin Z, Liu Z et al (2019) Structural basis for reversible amyloids of hnRNPA1 elucidates their role in stress granule assembly. Nat Commun 10:2006

18. Hergesheimer RC, Chami AA, de Assis DR, Vourc'h P, Andres CR, Corcia $P$ et al (2019) The debated toxic role of aggregated TDP-43 in amyotrophic lateral sclerosis: a resolution in sight? Brain 142:1176-1194

19. Hilgers V (2015) Alternative polyadenylation coupled to transcription initiation: Insights from ELAV-mediated 3' UTR extension. RNA Biol 12:918-921

20. Hinman MN, Lou H (2008) Diverse molecular functions of $\mathrm{Hu}$ proteins. Cell Mol Life Sci 65:3168-3181

21. Ho R, Workman MJ, Mathkar P, Wu K, Kim KJ, O'Rourke JG et al (2021) Cross-comparison of human iPSC motor neuron models of familial and sporadic ALS reveals early and convergent transcriptomic disease signatures. Cell Syst 12:159-175.e9

22. Ince-Dunn G, Okano HJ, Jensen KB, Park W-Y, Zhong R, Ule J et al (2012) Neuronal Elav-like (Hu) proteins regulate RNA splicing and abundance to control glutamate levels and neuronal excitability. Neuron 75:1067-1080 
23. Johnson JO, Pioro EP, Boehringer A, Chia R, Feit H, Renton AE et al (2014) Mutations in the Matrin 3 gene cause familial amyotrophic lateral sclerosis. Nat Neurosci 17:664-666

24. Kakumoto K, Miyazaki T, Watanabe M, Darnell RB, Okano H, Okano H (2010) The maintenance of Purkinje axons by HuC in adult cerebellum. Neurosci Res 68:e61

25. Kakumoto K, Miyazaki T, Watanabe M, Darnell R, Okano H, Okano H (2011) The maintenance of Purkinje axons by an RNA-binding protein $\mathrm{HuC}$ in adult cerebellum. Neurosci Res 71:e95-e96

26. Kapeli K, Martinez FJ, Yeo GW (2017) Genetic mutations in RNA-binding proteins and their roles in ALS. Hum Genet 136:1193-1214

27. Kapeli K, Pratt GA, Vu AQ, Hutt KR, Martinez FJ, Sundararaman B et al (2016) Distinct and shared functions of ALS-associated proteins TDP-43, FUS and TAF15 revealed by multisystem analyses. Nat Commun 7:12143

28. Kiernan MC, Vucic S, Cheah BC, Turner MR, Eisen A, Hardiman $\mathrm{O}$ et al (2011) Amyotrophic lateral sclerosis. Lancet 377:942-955

29. Kim HJ, Kim NC, Wang Y-D, Scarborough EA, Moore J, Diaz $\mathrm{Z}$ et al (2013) Mutations in prion-like domains in hnRNPA2B1 and hnRNPA1 cause multisystem proteinopathy and ALS. Nature 495:467-473

30. King PH, Redden D, Palmgren JS, Nabors LB, Lennon VA (1999) $\mathrm{Hu}$ antigen specificities of ANNA-I autoantibodies in paraneoplastic neurological disease. J Autoimmun 13:435-443

31. Klim JR, Williams LA, Limone F, Guerra San Juan I, DavisDusenbery BN, Mordes DA et al (2019) ALS-implicated protein TDP-43 sustains levels of STMN2, a mediator of motor neuron growth and repair. Nat Neurosci 22:167-179

32. Krach F, Batra R, Wheeler EC, Vu AQ, Wang R, Hutt K et al (2018) Transcriptome-pathology correlation identifies interplay between TDP-43 and the expression of its kinase CK1E in sporadic ALS. Acta Neuropathol 136:405-423

33. Kwiatkowski TJ Jr, Bosco DA, Leclerc AL, Tamrazian E, Vanderburg CR, Russ C et al (2009) Mutations in the FUS/TLS gene on chromosome 16 cause familial amyotrophic lateral sclerosis. Science 323:1205-1208

34. Lu Y-L, Liu Y, Yoo AS (2020) Synergism between microRNA-124 and ELAVL3 drives neuronal gene upregulation during direct neuronal reprogramming of human fibroblasts. bioRxiv. 2020.03.25.006635

35. Luo N-A, Qu Y-Q, Yang G-D, Wang T, Li R-L, Jia L-T et al (2014) Post-transcriptional up-regulation of PDGF-C by $\mathrm{HuR}$ in advanced and stressed breast cancer. Int $\mathrm{J}$ Mol Sci 15:20306-20320

36. Ma WJ, Chung S, Furneaux H (1997) The Elav-like proteins bind to AU-rich elements and to the poly(A) tail of mRNA. Nucleic Acids Res 25:3564-3569

37. Mackenzie IRA, Bigio EH, Ince PG, Geser F, Neumann M, Cairns NJ et al (2007) Pathological TDP-43 distinguishes sporadic amyotrophic lateral sclerosis from amyotrophic lateral sclerosis with SOD1 mutations. Ann Neurol 61:427-434

38. Mejzini R, Flynn LL, Pitout IL, Fletcher S, Wilton SD, Akkari PA (2019) ALS genetics, mechanisms, and therapeutics: where are we now? Front Neurosci 13:1310

39. Melamed Z, López-Erauskin J, Baughn MW, Zhang O, Drenner K, Sun Y et al (2019) Premature polyadenylation-mediated loss of stathmin-2 is a hallmark of TDP-43-dependent neurodegeneration. Nat Neurosci 22:180-190

40. Mélé N, Berzero G, Maisonobe T, Salachas F, Nicolas G, Weiss $\mathrm{N}$ et al (2018) Motor neuron disease of paraneoplastic origin: a rare but treatable condition. J Neurol 265:1590-1599

41. Milani P, Amadio M, Laforenza U, Dell'Orco M, Diamanti L, Sardone V et al (2013) Posttranscriptional regulation of SOD1 gene expression under oxidative stress: Potential role of ELAV proteins in sporadic ALS. Neurobiol Dis 60:51-60

42. Neumann M, Bentmann E, Dormann D, Jawaid A, DeJesusHernandez M, Ansorge $\mathrm{O}$ et al (2011) FET proteins TAF15 and EWS are selective markers that distinguish FTLD with FUS pathology from amyotrophic lateral sclerosis with FUS mutations. Brain 134:2595-2609

43. Nicolas A, Kenna KP, Renton AE, Ticozzi N, Faghri F, Chia R et al (2018) Genome-wide analyses identify KIF5A as a novel ALS gene. Neuron 97:1268-1283.e6

44. Nolan M, Talbot K, Ansorge O (2016) Pathogenesis of FUSassociated ALS and FTD: insights from rodent models. Acta Neuropathol Commun 4:99

45. Ogawa Y, Kakumoto K, Yoshida T, Kuwako K-I, Miyazaki T, Yamaguchi J et al (2018) Elavl3 is essential for the maintenance of Purkinje neuron axons. Sci Rep 8:2722

46. Ogawa Y, Yamaguchi J, Yano M, Uchiyama Y, Okano HJ (2018) Elavl3 regulates neuronal polarity through the alternative splicing of an embryo-specific exon in AnkyrinG. Neurosci Res 135:13-20

47. Okano HJ, Darnell RB (1997) A hierarchy of Hu RNA binding proteins in developing and adult neurons. J Neurosci 17:3024-3037

48. Rabin SJ, Kim JMH, Baughn M, Libby RT, Kim YJ, Fan Y et al (2010) Sporadic ALS has compartment-specific aberrant exon splicing and altered cell-matrix adhesion biology. Hum Mol Genet 19:313-328

49. Ravits J, Laurie P, Fan Y, Moore DH (2007) Implications of ALS focality: rostral-caudal distribution of lower motor neuron loss postmortem. Neurology 68:1576-1582

50. Rosa Ma X, Prudencio M, Koike Y, Vatsavayai SC, Kim G, Harbinski F et al (2021) TDP-43 represses cryptic exon inclusion in FTD/ALS gene UNC13A. https://doi.org/10.1101/2021. 04.02 .438213

51. Rotem N, Magen I, Ionescu A, Gershoni-Emek N, Altman T, Costa CJ et al (2017) ALS along the axons-expression of coding and noncoding RNA differs in axons of ALS models. Sci Rep 7:44500

52. De Santis R, Alfano V, de Turris V, Colantoni A, Santini L, Garone MG et al (2019) Mutant FUS and ELAVL4 (HuD) aberrant crosstalk in amyotrophic lateral sclerosis. Cell Rep 27:3818$3831 . e 5$

53. Scheckel C, Drapeau E, Frias MA, Park CY, Fak J, ZuckerScharff I et al (2016) Regulatory consequences of neuronal ELAV-like protein binding to coding and non-coding RNAs in human brain. Elife. https://doi.org/10.7554/eLife.10421

54. Taylor JP, Brown RH Jr, Cleveland DW (2016) Decoding ALS: from genes to mechanism. Nature 539:197-206

55. Ticozzi N, Vance C, Leclerc AL, Keagle P, Glass JD, McKenna-Yasek D et al (2011) Mutational analysis reveals the FUS homolog TAF15 as a candidate gene for familial amyotrophic lateral sclerosis. Am J Med Genet B Neuropsychiatr Genet 156B:285-290

56. Urdaneta EC, Vieira-Vieira $\mathrm{CH}$, Hick $\mathrm{T}$, Wessels $\mathrm{H}-\mathrm{H}$, Figini D, Moschall R et al (2019) Purification of cross-linked RNAprotein complexes by phenol-toluol extraction. Nat Commun 10:990

57. Vanden Broeck L, Callaerts P, Dermaut B (2014) TDP-43-mediated neurodegeneration: towards a loss-of-function hypothesis? Trends Mol Med 20:66-71

58. Wijesekera LC, Leigh PN (2009) Amyotrophic lateral sclerosis. Orphanet J Rare Dis 4:3

59. Wu S, Wang Y, Lin L, Si X, Wang T, Zhong X et al (2014) Protease $2 \mathrm{~A}$ induces stress granule formation during coxsackievirus B3 and enterovirus 71 infections. Virol J 11:192 
60. Zhang Z, So K, Peterson R, Bauer M, Ng H, Zhang Y et al (2019) Elav-mediated exon skipping and alternative polyadenylation of the Dscam1 gene are required for axon outgrowth. Cell Rep 27:3808-3817.e7

61. Zhao M, Kim JR, van Bruggen R, Park J (2018) RNA-binding proteins in amyotrophic lateral sclerosis. Mol Cells 41:818-829

62. Zhao W, Zhao J, Hou M, Wang Y, Zhang Y, Zhao X et al (2014) HuR and TIA1/TIAL1 are involved in regulation of alternative splicing of SIRT1 pre-mRNA. Int J Mol Sci 15:2946-2958
Publisher's Note Springer Nature remains neutral with regard to jurisdictional claims in published maps and institutional affiliations. 